\title{
Skyrmionics in sight
}

\section{The nucleation and manipulation of individual skyrmions in magnetic nanostructures will be essential in any future skyrmionic device.}

Magnetic skyrmions are swirling nanoscale spin structures that are stable against perturbations and could potentially be used to create novel memory devices. The structures are also of fundamental interest because they have led, for example, to the discovery of intriguing topological phenomena. In skyrmionic memory devices, information could be encoded in the magnetic configuration and transferred by pushing the spin textures along nanostructures by means of spin-polarized currents. Compared with magnetic domain walls, which have also been proposed for magnetic storage applications, skyrmions can be moved by much lower current densities ${ }^{1}$, suggesting that the devices would have lower power consumption and less critical energy dissipation issues. Moreover, skyrmions can be as small as a few nanometres across and could potentially provide an ultrahigh information-storage density.

On page 899 of this issue, Naoto Nagaosa and Yoshinori Tokura review recent advances in the field ${ }^{2}$. Skyrmions were first observed only a few years ago, but have now been observed in various magnetic materials, including both bulk and thin-film samples. After a comprehensive account of these observations, Nagaosa and Tokura examine the topological properties and the current-induced dynamics of skyrmions, and discuss the prospects for applications in skyrmionics.

Significant challenges still need to be overcome before skyrmionic devices become a reality ${ }^{3}$. There are, for example, issues related to material properties: the skyrmion phase has been observed only up to temperatures of $250 \mathrm{~K}$ (ref. 4), and in most cases can only be stabilized by an external magnetic field. Furthermore, most reported observations have been of lattices of skyrmions in thin films, but it is likely that skyrmionic devices will require individual skyrmions to be nucleated and controllably propagated in nanostructured thin-films.

Considerable progress has already been made in addressing these issues, particularly in 2013. Computational studies ${ }^{5,6}$ have examined current-induced skyrmion motion in confined geometries, and the nucleation of skyrmions in nanowires and nanodisks with spinpolarized currents ${ }^{7}$; individual skyrmions have been nucleated using a spin-polarized current from a scanning tunnelling microscope $e^{8}$; and skyrmion lattices have been observed in MnSi nanowires ${ }^{9}$. Among other advances, the coming year could perhaps see an experimental demonstration of current-induced nucleation and propagation of individual skyrmions in nanowires.

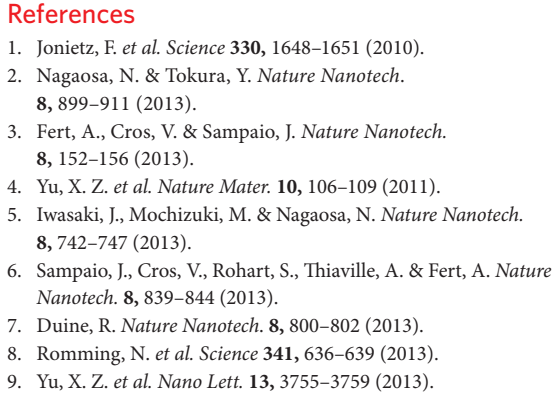

\section{An organic response}

\section{Researchers in organic spintronics consider a more structured approach to the field.}

In our September 2013 issue, we published a Commentary that highlighted a degree of stagnation in the field of organic spintronics ${ }^{1}$. This stagnation was attributed primarily to the proliferation of models that were conceived to explain specific sets of data but are not valid in general. We, and the authors, felt that the publication of the article could provide an opportunity for researchers in the field to reflect on the situation, and we solicited replies ${ }^{2}$.

We would like to thank all of our readers who provided feedback, and, in this issue, we are publishing six of the comments we received (page 884). The Correspondences we have selected were chosen because we felt they provided a notable contribution to the discussion. One of the comments is a technical reply from the authors of a paper that was mentioned extensively in the Commentary. All those who wrote to us broadly agree with the apparent stagnation of the field, although the general feeling seems to be that testing the models through spectroscopy - the main proposal in the Commentary - would not, alone, solve the problem. Combining spectroscopy and transport experiments, standardizing data and designing models starting from the molecular level are among the additional methods proposed. Finally, the simple use of logical reasoning is highlighted as a powerful tool when spectroscopy alone could lead to the wrong conclusions.

These Correspondences do not, of course, settle the debate. However, from the responses we have received, it seems that the community has found the exercise useful. It is likely that other fields would benefit from such a process - stepping back and reflecting on where to go next - and we shall certainly consider repeating the process for other areas in the future.

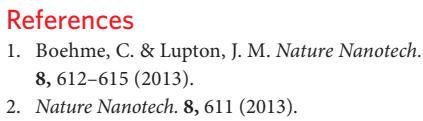

